\title{
Trace Proteinuria and the Incidence of Overt Proteinuria After Five Years: Results of the Kanagawa Investigation of the Total Checkup Data From the National Database-5 (KITCHEN-5)
}

\author{
Kei Nakajimaa, b, c, d, Ryoko Higuchia ${ }^{a}$, Kaori Mizusawa ${ }^{a}$
}

\begin{abstract}
Background: Trace proteinuria (TrP), which is usually defined as \pm by dipstick urinalysis, is considered as normal and of limited clinical significance. However, the relationship between $\mathrm{TrP}$ and overt proteinuria (OP) $(\geq+1)$ in the future is unknown. Therefore, we investigated the association between $\mathrm{TrP}$ and future incidence of OP in a community-based cohort study.
\end{abstract}

Methods: TrP detected during the initial 2 years, which was classified into transient $\operatorname{TrP}$ (once/2 years) (T-TrP) and recurrent $\operatorname{TrP}$ (twice/2 years) (R-TrP); and the incidence of OP after 5 years were investigated in 292,257 general Japanese people aged 40 - 68 years who attended checkups. To determine TrP and OP, dipstick urinalysis was conducted with visual reading (VR) by medical staff or automated reading $(A R)$ using a machine reader.

Results: Overall, T-TrP and R-TrP were observed in 24,782 (8.5\%) and 3,767 (1.3\%) subjects, respectively. Both types of TrP were prevalent in the detection with AR than VR. The prevalences of T-TrP and $\mathrm{R}-\mathrm{Tr} \mathrm{P}$ showed $\mathrm{J}$-shaped relationships against baseline body mass index (BMI), regardless of sex and BMI categories. The incident of OP after 5 years was larger (around 10\%) in R-TrP than T-TrP (around 5\%): approximately two times. Logistic regression analysis showed that T-TrP and R-TrP were significantly associated with OP, even after adjustment for relevant confounding factors including age, sex, and

Manuscript submitted June 27, 2020, accepted July 23, 2020

Published online August 15, 2020

aSchool of Nutrition and Dietetics, Faculty of Health and Social Services, Kanagawa University of Human Services, 1-10-1 Heisei-cho, Yokosuka, Kanagawa 238-8522, Japan

${ }^{b}$ Graduate School of Health Innovation, Kanagawa University of Human Services, Research Gate Building Tonomachi 2-A, 3-25-10 Tonomachi, Kawasaki, Kanagawa 210-0821, Japan

'Department of Endocrinology and Diabetes, Saitama Medical Center, Saitama Medical University, 1981 Kamoda, Kawagoe, Saitama 350-8550, Japan ${ }^{\mathrm{d} C}$ Corresponding Author: Kei Nakajima, School of Nutrition and Dietetics, Faculty of Health and Social Services, Kanagawa University of Human Services, 1-10-1 Heisei-cho, Yokosuka, Kanagawa 238-8522, Japan.

Email: nakajima-rsh@kuhs.ac.jp

doi: https://doi.org/10.14740/jocmr4274
BMI (odds ratios (95\% confidence intervals (CIs)): 2.77 (2.60 - 2.95) and 4.85 (4.34 - 5.43)), which were not largely altered when subanalysis was conducted according to men and women, non-obesity and obesity, or AR and VR. In all analysis above, the odds ratios $(95 \%$ CIs) of R-TrP for OP were higher than T-TrP.

Conclusions: Our findings suggest that $\operatorname{Tr} \mathrm{P}$, particularly R-TrP, is substantially associated with the future incidence of OP, which may be independent of confounding factors and the methods detecting TrP.

Keywords: Trace proteinuria; Dipstick urinalysis; Overt proteinuria; Body mass index; Visual reading; Automated reading

\section{Introduction}

Kidney damage usually begins as an incident of proteinuria, which is most often assessed as microalbuminuria in the clinical setting for early detection of kidney disease [1, 2]. However, in apparently healthy community dwellers, who are supposed to undergo annual checkups for managing their health, measurement of microalbuminuria is unfeasible because of its cost and it is time consuming. In an ordinary health checkup, proteinuria defined as $\geq+1$ is measured using dipstick urinalysis, which is considered as unable to detect microalbuminuria [3].

To date, clinical significance of trace proteinuria $(\mathrm{TrP})$, which is assessed as \pm by dipstick urinalysis, is generally classified as normal and of limited clinical significance [3-6]. In spite of limited evidence, several studies have suggested that $\operatorname{TrP}$ usually indicates microalbuminuria $[4,7]$, and that a large portion of microalbuminuria (albumin/creatinine ratio $(30-300 \mathrm{mg} / \mathrm{g})$ ) overlaps with $\mathrm{TrP}$ [6]. However, the association between $\operatorname{TrP}$ and overt proteinuria (OP) $(\geq+1)$, which is an established marker of chronic kidney disease $[3,5]$, is unknown.

Therefore, we investigated the association between $\operatorname{TrP}$ detected during the initial 2 years and the incidence of OP after 5 years in a community-based cohort study. The cohort comprised 292,257 healthy people who did not have OP during the initial 2 years. Because proteinuria including TrP can transiently result from various physiological conditions [1, 
3], we classified $\operatorname{TrP}$ into transient $\operatorname{Tr} P$ (positive once in 2 years) (T-TrP) and recurrent $\operatorname{TrP}$ (positive twice in 2 years) (R-TrP). Additionally, we investigated the difference in the detection rate for TrP between visual reading (VR) by medical staff and automated reading (AR) using a machine, because this difference can affect the detection rate of TrP.

\section{Materials and Methods}

\section{Study design}

Our project was a composite study that consisted of secondary use of health checkup data in Japan, which is termed the Kanagawa Investigation of the Total Checkup Data from the National Database (KITCHEN). This original study aimed to determine factors associated with cardiometabolic diseases. The study design is described in detail elsewhere [8]. Since 2008, all people living in Japan aged 40 - 74 years are supposed to undergo a yearly specific health checkup managed by the Ministry of Health, Labour and Welfare (MHLW) [9]. The study protocol was approved by the Ethics Committee of Kanagawa University of Human Services (No.10-43) and MHLW in Japan (No. 121). We received digitally recorded anonymous data regarding people living in Kanagawa Prefecture from the MHLW as part of its nationwide program, which involves the provision of medical data to third parties. To protect against the identification of specific individuals, their ages were already categorized as 40 - 44, 45 - 49, 50 - 54, 55 - 59, 60 - 64, and 65 - 69 years. In this study, to evaluate subject age as a single numeric value, we transformed the age groups into substituted ages (s-age), corresponding to the median for each age group (42, 47, 52, 57, 62, and 67 years, respectively). This study was conducted in compliance with the ethical standards of the responsible institution on human subjects as well as with the Helsinki Declaration.

\section{Subjects}

This cohort study used data of people who attended checkups three times during 6 years: April 2008 to March 2010 (initial 2 years) and April 2014 to March 2015. Participants who reported having kidney disease during the initial 2 years from April 2008 to March 2010 were excluded. Subjects with OP during the initial 2 years were also excluded. Consequently, 292,257 subjects aged 40 - 68 years with complete data (men, 58.0\%) were enrolled in the study. However, individuals at moderate to severe conditions were unlikely to be involved in this study because healthcare stuff in checkup institutions advised such people to go a hospital or clinic immediately.

\section{Measurements of clinical parameters}

Measurements were conducted in the morning after an overnight fast. Body mass index (BMI) was calculated as weight $(\mathrm{kg})$ divided by the square of height $\left(\mathrm{m}^{2}\right)$. Clinical parameters were measured with internal and external standards, as instructed by the Ministry of Health, Labour and Welfare [9]. Dipstick urinalysis was conducted with VR by medical staff or by AR using a machine reader with reflectance photometry. The grade of proteinuria was classified as five grades: none $(-)$, trace $( \pm),+1,+2$, and $\geq+3$. We classified TrP into T-TrP (positive once in 2 years) and R-TrP (positive twice in 2 years). Because OP and TrP showed a J-shaped relationship against BMI in previous studies $[10,11]$, the relationship between two types of $\operatorname{TrP}$ and BMI were examined in this study.

Unfortunately, the data of estimated glomerular filtration rate (eGFR) and measurements related to urine specimens were unavailable in our database because this checkup had started for the screening of metabolic syndrome [8], although it is well known at present time that metabolic syndrome and cardiovascular disease are closely associated with impaired kidney function $[12,13]$.

\section{Statistical analysis}

Data are expressed as the mean \pm standard deviation (SD) or median (interquartile range). Continuous and categorical variables were analyzed by analysis of variance (ANOVA) and the $\chi^{2}$ test, respectively. A trend for $\mathrm{T}-\mathrm{TrP}$ and $\mathrm{R}-\mathrm{TrP}$ was tested by the Cochran-Armitage test. The Mantel-Haenszel test was used to investigate the effect of different interpretations (VR alone, VR/AR, and AR alone) of the percentages of two types of TrP. Logistic regression models were used to examine the associations between $\mathrm{T}-\mathrm{TrP}$ and R-TrP and OP, with adjustment for relevant confounding factors, yielding odds ratios (ORs) and 95\% confidence intervals (CIs). Confounding factors were chosen based on biological plausibility, but not with stepwise procedure. Statistical analyses were performed using SAS-Enterprise Guide (SAS-EG 7.1) in the SAS system, version 9.4 (SAS Institute, Cary, NC, USA). P $<0.05$ was considered significant.

\section{Results}

Baseline characteristics of the subjects according to TrP categories are shown in Table 1 . The prevalence of T-TrP $(8.5 \%)$ was much higher than that of R-TrP $(1.3 \%)\left(\mathrm{P}<0.0001, \chi^{2}\right.$ test). As shifted from absence of TrP to T-TrP and R-TrP, age, BMI, systolic blood pressure, serum triglyceride levels, and HbA1c levels were significantly increased, whereas serum high-density lipoprotein cholesterol levels were decreased. Simultaneously, the prevalence of men, pharmacotherapies (hypertension, diabetes, and dyslipidemia), current smokers, and daily alcohol drinkers was increased, although the prevalence of habitual exercisers was not. Half of dipstick urinalysis was interpreted with AR alone (49.8\%) and 39.9\% was interpreted with VR alone. The proportions of T-TrP and R-TrP were significantly higher when they were measured with AR alone compared with VR alone (Mantel-Haenszel test, $\mathrm{P}<0.0001$ ). The relationship between the prevalence of $\mathrm{T}-\mathrm{TrP}$ and $\mathrm{R}-\mathrm{TrP}$ with BMI categories showed a J-shaped curve with the bottom 
Table 1. Baseline Characteristics of the Subjects According to T-TrP and R-TrP

\begin{tabular}{|c|c|c|c|}
\hline & Absence of TrP & T-TrP & R-TrP \\
\hline $\mathrm{N}(\%$ in total $)$ & $263,708(90.2)$ & $24,782(8.5)$ & $3,767(1.3)$ \\
\hline \multicolumn{4}{|l|}{ Methods of detection for proteinuria } \\
\hline VR/AR for each year, n (\%) & $26,886(89.0)$ & $3,040(10.1)$ & $300(1.0)$ \\
\hline AR for both years, $\mathrm{n}(\%)$ & $129,058(88.8)$ & $13,925(9.6)$ & $2,417(1.7)^{\mathrm{a}}$ \\
\hline Body mass index $\left(\mathrm{kg} / \mathrm{m}^{2}\right)$ & $22.8 \pm 3.2$ & $23.2 \pm 3.5$ & $23.6 \pm 3.6$ \\
\hline Systolic blood pressure (mm Hg) & $123 \pm 16.6$ & $124 \pm 17.2$ & $125 \pm 17.7$ \\
\hline Triglycerides (mg/dL) & $95(67-140)$ & $98(69-144)$ & $106(72-155)$ \\
\hline High-density lipoprotein cholesterol (mg/dL) & $64.3 \pm 16.9$ & $63.1 \pm 17.0$ & $61.4 \pm 17.0$ \\
\hline \multicolumn{4}{|l|}{ Pharmacotherapy for } \\
\hline Diabetes, n (\%) & $5,579(2.1)$ & $792(3.2)$ & $165(4.4)$ \\
\hline Dyslipidemia, n (\%) & $21,682(8.2)$ & $2,284(9.2)$ & $383(10.2)$ \\
\hline Past history of cardiovascular disease, n (\%) & $6,935(2.6)$ & $805(3.3)$ & $176(4.7)$ \\
\hline Current smokers, n (\%) & $64,389(24.4)$ & $6,698(27.0)$ & $1,155(30.7)$ \\
\hline Habitual exercisers, $\mathrm{n}(\%)^{\mathrm{b}}$ & $77,174(29.3)$ & $7,194(29.0)$ & $1,069(28.4)$ \\
\hline Daily alcohol drinkers, $\mathrm{n}(\%)^{\mathrm{c}}$ & $78,399(29.7)$ & $7,460(30.1)$ & $1,214(32.2)$ \\
\hline
\end{tabular}

Data are presented as mean $\pm \mathrm{SD}$, median (interquartile range), or number (\%). All $\mathrm{P}$ values, except for exercise and alcohol drinkers, determined by ANOVA (continuous variables), and the $x^{2}$ and Cochran-Armitage test (categorical variables), are $<0.0001$, respectively. ${ }^{a}$ Methods of detection for TrP, Mantel-Haenszel test $(P<0.0001)$. ${ }^{b}$ Exercise of sweating lightly for over 30 min per session, two times weekly, $X^{2}$ test, $P=0.38$; CochranArmitage test, $P=0.18 .{ }^{c} X^{2}$ test, $P=0.002$; Cochran-Armitage test, $P=0.003$. TrP: trace proteinuria; $T$-TrP: transient trace proteinuria; $R$-TrP: recurrent trace proteinuria; s-age: substituted age; VR: visual reading; AR: automated reading using a machine; SD: standard deviation; ANOVA: analysis of variance.

of the curve at a BMI of approximately $20.0 \mathrm{~kg} / \mathrm{m}^{2}$ (Fig. 1). The T-TrP was higher than R-TrP, regardless of sex and BMI categories.

Table 2 shows the incidents of OP after 5 year according to types of $\operatorname{TrP}$ and the sub-groups. Overall, the incident of OP after 5 years was larger (around 10\%) in R-TrP than T-TrP (around 5\%): approximately two times. Subsequently, logistic regression analysis showed that $\mathrm{T}-\mathrm{TrP}$ and $\mathrm{R}-\mathrm{TrP}$ were significantly associated with OP after 5 years, even after adjustment for relevant confounders, including baseline BMI (Table 2). Associations after full adjustment were not perceivably altered when subjects were divided into three groups who had TrP interpreted with different methods (VR alone, VR/AR, and AR alone), non-obese subjects (BMI $<25.0 \mathrm{~kg} / \mathrm{m}^{2}$ ) and obese subjects $\left(\mathrm{BMI} \geq 25.0 \mathrm{~kg} / \mathrm{m}^{2}\right)$, or men and women.

\section{Discussion}

This large epidemiological study showed that TrP, especially $\mathrm{R}-\mathrm{TrP}$, was significantly associated with the incidence of OP after 5 years, independently of relevant confounders and the methods detecting TrP. Our results also suggest that the pro- portion of both types of TrP may be affected to a certain extent by the detection methods for proteinuria. This finding deserves further study to confirm whether the observed difference is due to underestimation by VR or overestimation by AR. The observed relationship of T-TrP and R-TrP against BMI is similar to that of OP [10] and consistent with our previous crosssectional study [11]. Although this study does not individually demonstrate a transformation from TrP to OP in the time course, the current results strongly suggest that $\operatorname{TrP}$, especially $\mathrm{R}-\mathrm{TrP}$, may eventually result in OP. Because measurement of microalbuminuria is time consuming and expensive, TrP may be a surrogate marker for early kidney damage in individuals who are unlikely to have microalbuminuria measured. However, this could increase the false-positive rate for detecting early kidney damage [6]. Therefore, R-TrP may be preferable than $\mathrm{T}-\mathrm{TrP}$ as a target for intervention because of the reduction in false-positive rate. Consistently, the incident of OP in R-TrP was approximately two times higher compared with that in TTrP. Clinical studies in recent years [14-16] have shown that TrP may be a risk factor for metabolic syndrome and increased all-cause mortality. Therefore, not only OP, but also TrP, may have clinical significance for cardiometabolic conditions, including kidney disease. 


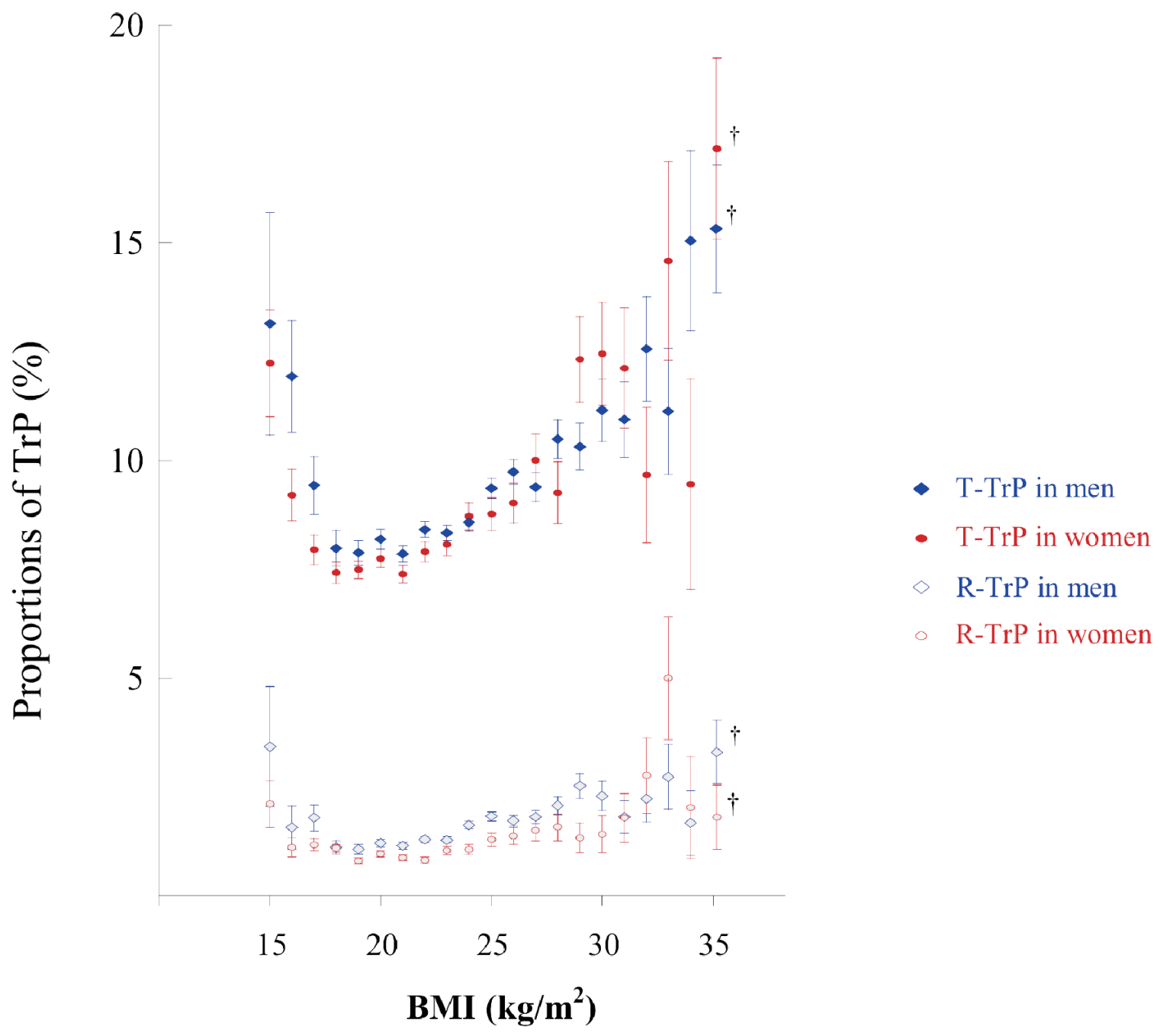

Figure 1. Proportion of subjects with TrP according to BMI categories. The circle in the middle of each bar shows the mean percentage of subjects with $\operatorname{TrP}$ ( \pm on dipstick urinalysis) according to BMI intervals $\left(1.0 \mathrm{~kg} / \mathrm{m}^{2}\right)$. This percentage was calculated as the number of subjects with TrP/number of subjects in each BMI group $\times 100$ for each BMI interval $\left(1.0 \mathrm{~kg} / \mathrm{m}^{2}\right.$ intervals $)$. The vertical bar represents the standard error when $\operatorname{TrP}$ is 1 and non- $\operatorname{TrP}$ is 0 . ${ }^{\dagger} \mathrm{BMI}$ from 35.0 to $50.0 \mathrm{~kg} / \mathrm{m}^{2}$. BMI: body mass index; TrP: trace proteinuria; T-TrP: transient TrP; R-TrP: recurrent TrP.

Some limitations of our study should be mentioned. First, eGFR and measurements related to urine specimens, including urine gravity, hematuria, and bacteriuria, were unavailable in this study. Then, kidney function and the conditions of urine sampling were not considered in this study. However, $\operatorname{TrP}$ may be frequently observed in an early stage of kidney disease [4, 7]. In addition, it has been shown that GFR is well preserved in patients with microalbuminuria [17]. Therefore, adjustment for eGFR might not largely affect the association between $\operatorname{TrP}$ and OP, although this issue deserves further study. Second, although interpretation of AR in dipstick urinalysis may be more sensitive than VR for TrP, similar to OP, this warrants confirmation in additional studies because conflicting results remain [18-20].

In conclusion, our results suggest that $\mathrm{Tr} P$, particularly $\mathrm{R}-\mathrm{TrP}$, is substantially associated with the future incidence of OP, which may be independent of confounding factors and the methods detecting TrP. However, further study including eGFR and urine specimens are needed to confirm current results and underlying mechanism.

\section{Acknowledgments}

The submission of this manuscript was approved by Japanese Ministry of Health, Labor and Welfare in August 2020. We thank Ellen Knapp, PhD, from Edanz Group for editing a draft of this manuscript.

\section{Financial Disclosure}

None to declare. 
Table 2. Odds Ratios (ORs) (95\% Confidence Intervals) of T-TrP and R-TrP for the Incident of OP After Five Years

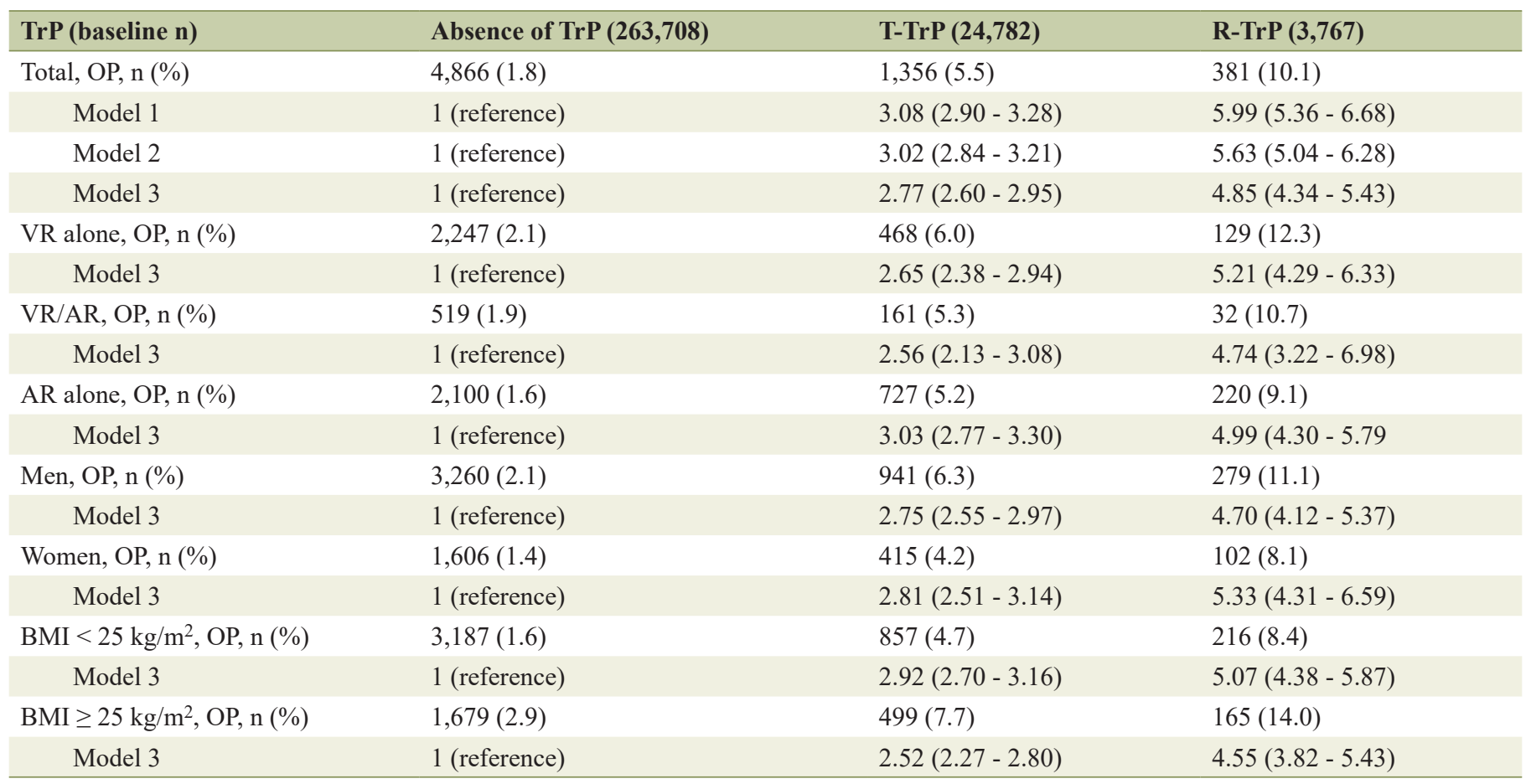

Model 1: unadjusted. Model 2: adjusted for age and sex. Model 3: model 2 plus adjustment for current smoking (versus non-smoking), daily alcohol consumption (versus infrequent/no alcohol consumption), regular exercise (versus no regular exercise), pharmacotherapy (for hypertension, diabetes, or dyslipidemia), body mass index, systolic blood pressure, triglycerides, high-density lipoprotein cholesterol, HbA1c (as continuous variables), and past history of cardiovascular disease (versus no history). TrP: trace proteinuria; T-TrP: transient trace proteinuria; R-TrP: recurrent trace proteinuria; OP: overt proteinuria; AR: automated reading; VR: visual reading. All P values of ORs of T-TrP and R-TrP for OP are $<0.0001$.

\section{Conflict of Interest}

None of the authors have any potential conflict of interest.

\section{Informed Consent}

Not applicable. We received the digitally recorded non-distinguishing anonymous data from the MHLW in August 2017. We have opened the protocol of our study to the public on our university homepage, which was updated in October 2017.

\section{Author Contributions}

$\mathrm{KN}$ contributed to the study design and the interpretation of the initial analysis. $\mathrm{RH}$ and $\mathrm{KM}$ contributed to the preparation and management of data used in this study. $\mathrm{KN}$ and $\mathrm{RH}$ conducted the data analysis and the discussion of the literature. $\mathrm{KN}$ prepared the first draft of the manuscript, and all authors read and edited the manuscript.

\section{Data Availability}

Any inquiries regarding supporting data availability of this study should be directed to the corresponding author.

\section{References}

1. Bergstein JM. A practical approach to proteinuria. Pediatr Nephrol. 1999;13(8):697-700.

2. Currie G, Delles C. Proteinuria and its relation to cardiovascular disease. Int J Nephrol Renovasc Dis. 2013;7:1324.

3. Levey AS, Coresh J, Balk E, Kausz AT, Levin A, Steffes MW, Hogg RJ, et al. National Kidney Foundation practice guidelines for chronic kidney disease: evaluation, classification, and stratification. Ann Intern Med. 2003;139(2):137-147.

4. Sam R, Shaykh MS, Pegoraro AA, Khalili V, Hristea I, Singh AK, Arruda JA, et al. The significance of trace proteinuria. Am J Nephrol. 2003;23(6):438-441.

5. Lamb EJ, MacKenzie F, Stevens PE. How should proteinuria be detected and measured? Ann Clin Biochem. 2009;46(Pt 3):205-217.

6. Wen CP, Yang YC, Tsai MK, Wen SF. Urine dipstick to detect trace proteinuria: an underused tool for an underappreciated risk marker. Am J Kidney Dis. 2011;58(1):1-3.

7. Konta T, Hao Z, Takasaki S, Abiko H, Ishikawa M, Takahashi T, Ikeda A, et al. Clinical utility of trace proteinuria for microalbuminuria screening in the general population. 
Clin Exp Nephrol. 2007;11(1):51-55.

8. Nakajima K, Iwane T, Higuchi R, Shibata M, Takada $\mathrm{K}$, Uda J, Anan M, et al. Kanagawa investigation of the total check-up data from the national database (KITCH$\mathrm{EN})$ : protocol for data-driven population-based repeated cross-sectional and 6-year cohort studies. BMJ Open. 2019;9(2):e023323.

9. Program of standard checkup and health guidance (Ministry of Health, Labour and Welfare). https://www. mhlw.go.jp/file/06-Seisakujouhou-10900000-Kenkoukyoku/00 3.pdf.

10. Muneyuki T, Sugawara H, Suwa K, Oshida H, Saito M, Hori Y, Seta S, et al. A community-based cross-sectional and longitudinal study uncovered asymptomatic proteinuria in Japanese adults with low body weight. Kidney Int. 2013;84(6):1254-1261.

11. Nakajima K, Kanda E, Muneyuki T, Suwa K. Asymptomatic trace and overt proteinuria in high- and lowbody weight individuals: A preliminary report of communitybased epidemiological study. J Nephropathol. 2018;7:5156.

12. Singh AK, Kari JA. Metabolic syndrome and chronic kidney disease. Curr Opin Nephrol Hypertens. 2013;22(2):198-203.

13. Assadi F. The growing epidemic of chronic kidney disease: preventive strategies to delay the risk for progression to ESRD. Adv Exp Med Biol. 2019;1121:57-59.

14. Okada R, Yasuda Y, Tsushita K, Wakai K, Hamajima N, Matsuo S. Trace proteinuria by dipstick screening is as- sociated with metabolic syndrome, hypertension, and diabetes. Clin Exp Nephrol. 2018;22(6):1387-1394.

15. Kwon Y, Han K, Kim YH, Park S, Kim DH, Roh YK, Park YG, et al. Dipstick proteinuria predicts all-cause mortality in general population: A study of 17 million Korean adults. PLoS One. 2018;13(6):e0199913.

16. Han EN, Lee KB, Kim H, Hyun YY. Trace urine albumin and mortality: Kangbuk Samsung Health Study. Kidney Blood Press Res. 2018;43(3):951-958.

17. Mogensen CE, Hansen KW, Nielsen S, Pedersen MM, Rehling M, Schmitz A. Monitoring diabetic nephropathy: glomerular filtration rate and abnormal albuminuria in diabetic renal disease - reproducibility, progression, and efficacy of antihypertensive intervention. Am J Kidney Dis. 1993;22(1):174-187.

18. Waugh JJ, Bell SC, Kilby MD, Blackwell CN, Seed P, Shennan AH, Halligan AW. Optimal bedside urinalysis for the detection of proteinuria in hypertensive pregnancy: a study of diagnostic accuracy. BJOG. 2005;112(4):412417.

19. De Silva DA, Halstead AC, Cote AM, Sabr Y, von Dadelszen P, Magee LA. Urinary dipstick proteinuria testing: does automated strip analysis offer an advantage over visual testing? J Obstet Gynaecol Can. 2014;36(7):605612.

20. Correa ME, Cote AM, De Silva DA, Wang L, Packianathan P, von Dadelszen P, Magee LA. Visual or automated dipstick testing for proteinuria in pregnancy? Pregnancy Hypertens. 2017;7:50-53. 